\title{
Water and wastewater digital surveillance for monitoring and early detection of the COVID-19 hotspot: industry 4.0
}

\author{
A. N. Matheri ${ }^{1}$ (D) M. Belaid ${ }^{1}$. C. K. Njenga ${ }^{2} \cdot$ J. C. Ngila $^{3}$
}

Received: 13 December 2020 / Revised: 24 July 2021 / Accepted: 18 January 2022 / Published online: 7 February 2022

(C) Islamic Azad University (IAU) 2022

\begin{abstract}
There are a high number of COVID-19 cases per capita in the world that goes undetected including clinical diseases compatible with COVID-19. While the presence of the COVID-19 in untreated drinking water is possible, it is yet to be detected in the drinking-water supplies. COVID-19 viral fragments have been found in excrete, this call for wastewater monitoring and analysis (wastewater surveillance) of the potential health risk. This raises concern about the potential of the SARS$\mathrm{CoV}-2$ transmission via the water systems. The economic limits on the medical screening for the SARS-CoV-2 or COVID19 worldwide are turning to wastewater-based epidemiology as great potential tools for assessing and management of the COVID-19 pandemic. Surveillance and tracking of the pathogens in the wastewater are key to the early warning system and public health strategy monitoring of the COVID-19. Currently, RT-qPCR assays is been developed for SARS-CoV-2 RNA specimen clinical testing and detection in the water system. Convectional wastewater treatment methods and disinfection are expected to eradicate the SAR-CoV-2. Chlorine, UV radiation, ozone, chloramine is been used to inactivate and disinfect the water treatment system against the SARS-CoV-2. Water management and design of the water infrastructure require major changes to accommodate climate change, water cycle, reimaging of digitalization, infrastructure and privacy protection. The water digital revolution, biosensors and nanoscale, contact tracing, knowledge management can accelerate with disruption of the COVID-19 outbreak (water-health-digital nexus).
\end{abstract}

Keywords Epidemiology $\cdot$ Monitoring $\cdot$ Pathogens $\cdot$ SARS-CoV-2 RNA $\cdot$ RT-qPCR $\cdot$ Wastewater surveillance

\section{Introduction}

Severe acute respiratory syndrome coronavirus 2 (SARS$\mathrm{CoV}-2$ ) that caused the global pandemic of the coronavirus (COVID-19) emerged from Wuhan, China and spread globally. The outbreak was declared a public health emergence on 30th January 2020 by World Health Organization and on 11th February 2020 given a name of new coronavirus (COVID-19) (La Rosa et al. 2020). It is transmitted through

Editorial responsibility: Samareh Mirkia.

A. N. Matheri

anthonym@uj.ac.za

1 Department of Chemical Engineering, University of Johannesburg, Johannesburg, South Africa

2 United Nation Environmental Programme, Pretoria, South Africa

3 Department of Chemical Sciences, University of Johannesburg, Johannesburg, South Africa respiratory droplets (inhalation of the droplets) expelled from the mouth and noses of the infected person during speaking, coughing, sneezing and contaminated surfaces. There are a high number of COVID-19 cases per capita in the world that goes undetected including clinical diseases compatible with COVID-19. Mass testing on its own is not a solution to curbing the spread of SARS-COV-2 but part of the strategy when combine with immediate isolation, rapid diagnosis and tracking technology (contact tracking). The cost of contact tracking, testing and isolation are high in the short-term and long-term. This leads to disruption of the economic and social cost due to social distances that affect the communities, businesses and individuals. The measures are lethal or severe until the time the crisis is managed or through the intervention of the pharmaceutical (treatment or vaccination of the COVID-19) availability. Maintenance of the open economy and society while minimizing the population health crisis is important (Salathé et al. 2020). While the presence of the COVID-19 in untreated drinking water is possible, it is yet to be detected in the drinking-water 
supplies. COVID-19 is enveloped and thus less stable than the non-enveloped human enteric virus that is waterborne transmitted. COVID-19 viral fragments have been found in excrete, this call for wastewater monitoring and analysis (wastewater surveillance) of the potential health risk (W.H.O 2020). Figure 1 outlines and highlights the potential of the wastewater surveillance in the monitoring of the COVID-19 in water, wastewater system and public health (Farkas 2020).

The present study combined consideration on monitoring and mitigation, surveillance and tracking, economy, practicality, data capture and analysis in the water and wastewater surveillance when performing the population-wide screening for the SARS-CoV-2/COVID-19.

\section{Vulnerability to COVID-19}

The major concern underlying the potential burden to the COVID-19 in the low- and middle-income countries (LMICs) are vulnerability due to epidemiological (i.e. fatality rates due to the age, gender and underlying health conditions), transmission (i.e. hygiene infrastructure, population and housing density, urban mobility and immigration, unventilated and house confinement, high prices of the sanitisers and masks, shared toilets during a call of nature and lack of access to water, inadequate waste disposal leading to biohazards risks and social integration/distancing), high demand for the health system and availability of the intensive care, control measures of the social protection and economic package (Wilkinson 2020).

\section{Water, sanitation and hygiene (WASH) COVID-19 monitoring and mitigation}

Wastewater is a scarce resource that should be protected and reuse. The water in the sewer system acts as early warming to the outbreak of a disease in the urban population. COVID19 pandemic has highlighted the importance of proper management of water, sanitation and hygiene (WASH) and protection of human health (W.H.O 2020). Contaminated wastewater with pathogens and exposure to human beings and animals play a major role in the spread of the diseases. Surveillance and tracking of the pathogens in the wastewater is key to the early warning system (EWS) and public health strategy monitoring of the COVID-19 (Street et al. 2020). SARS-CoV-2 genetics matters can be detected in the gastro-intestinal waste of a COVID-19 patient and are transmitted through the faecal-oral route. The virus genetic have been detected in the wastewater treatment system and raising concern about the potential of the transmission route. The survival rate of the COVID-19 from the infective state in faecal matter is yet to be determined. According to (Pan et al. 2020), digestive systems were conformed from the patients of the COVID-19. Other symptoms included are respiratory, lack of appetite, diarrhoea, abdominal pain and vomiting. Convectional wastewater treatment methods and disinfection are expected to eradicate the SAR-CoV-2 but their high population and under-maintained wastewater treatment plants can contaminate the surroundings where the SAR-CoV-2 survive up to several days on untreated water and much longer in a lower temperature region. The prevention of water pollution from the collection, distribution, consumption is outlined by the World Health Organization

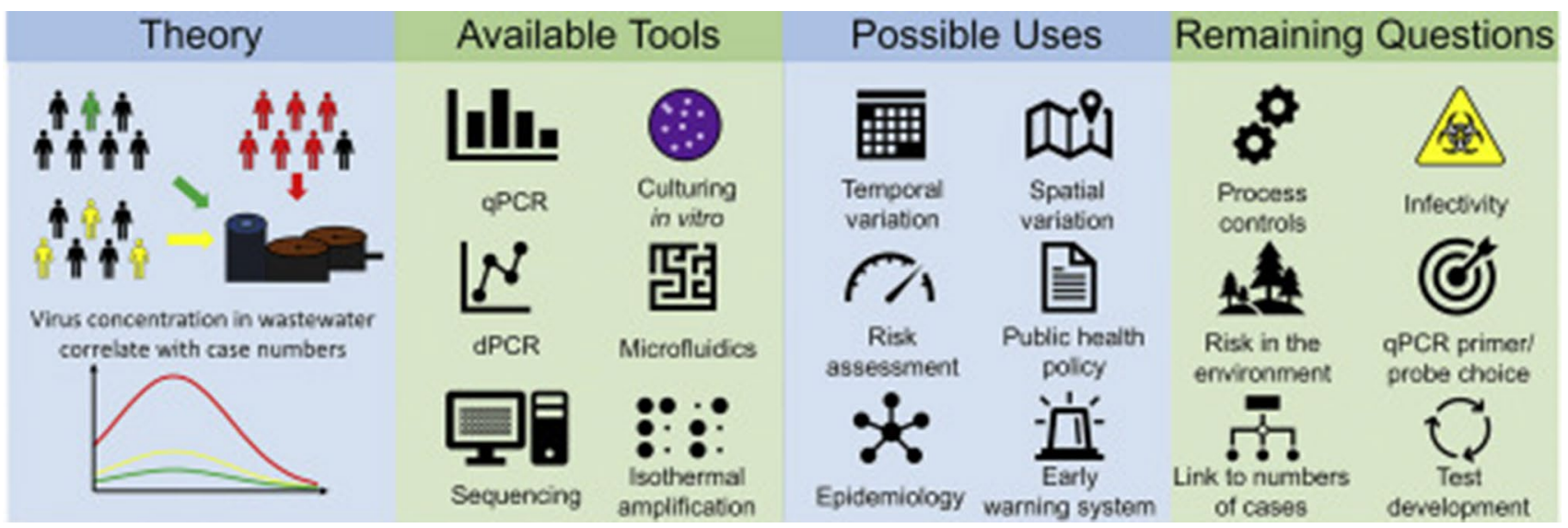

Fig. 1 Potential of the wastewater surveillance in monitoring for the COVID-19 in water, wastewater system and public health (Farkas et al. 2020) 


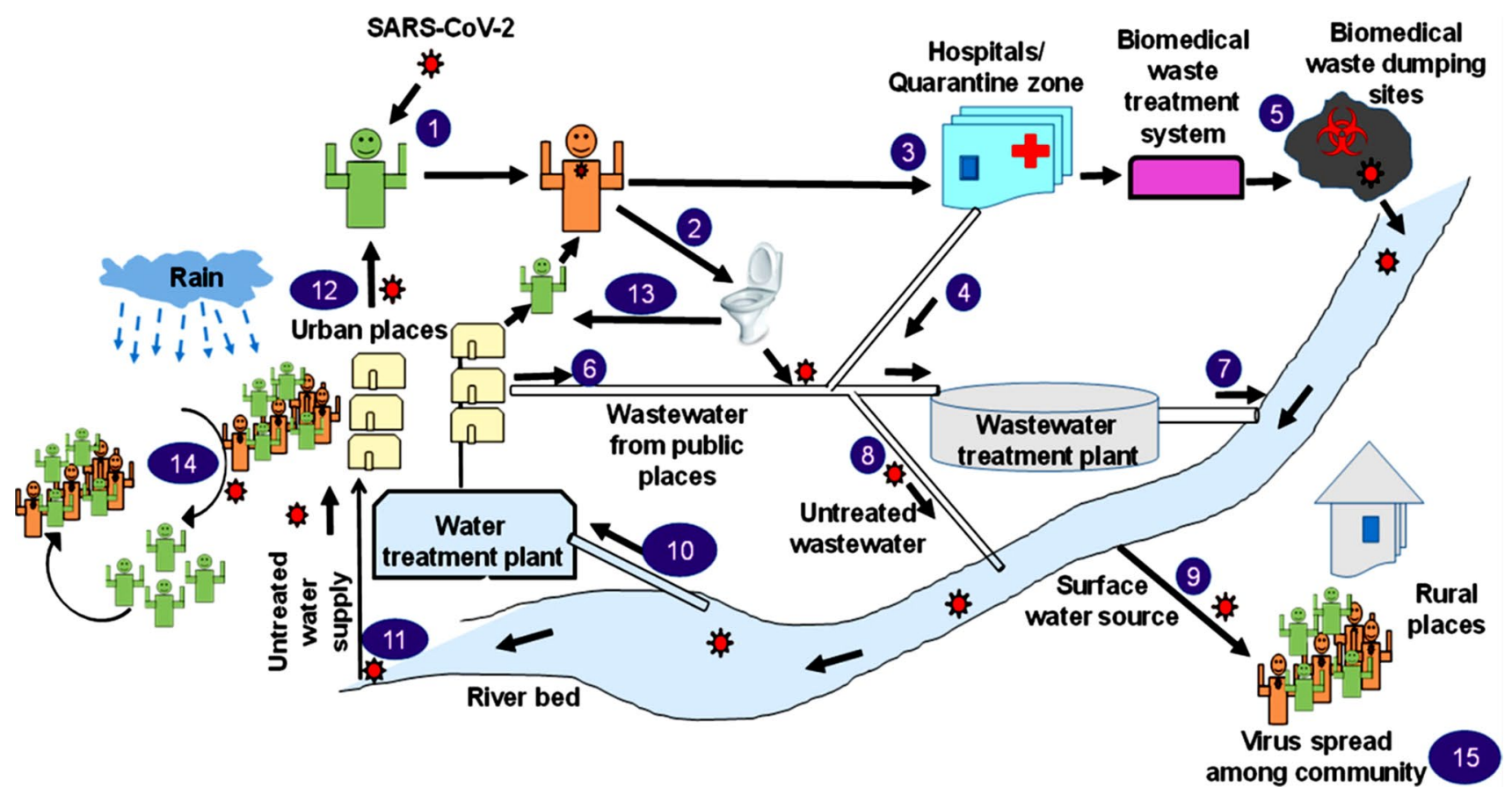

Fig. 2 COVID-19 possibility of contamination in the rural and urban water cycle with the potential of human exposure to the SARS-COV-2 (Bhowmick et al. 2020)

(WHO) for the resistance of the COVID-19 (W.H.O 2019). Figure 2 shows the COVID-19 possibility of contamination in the rural and urban water cycle with the potential of human exposure to the SARS-CoV-2 (Bhowmick et al. 2020).

The pathways indicates; (1) spread of the COVID-19 from animal to human being, (2) COVID-19 enters the wastewater systems using sewage system, (3) quarantine and hospitals centres, (4) contaminated wastewater flow to the wastewater treatment plant, (5) Mishandling and careless disposal of the biomedical waste contaminates the water system, (6) Wastewater generated in the public premises, (7) Discharge of the treated wastewater back to the receiving water, (8) Bypassing of the contaminated untreated wastewater into the water body, (9) Direct consumption of the contaminated water distribution network by rural communities, (10) Disinfected and treated of the contaminated wastewater at the WWTPs, (11) Direct consumption of the contaminated water distribution network by urban communities, (12) Increase in the COVID-19 cases due to contaminated water system in the urban region, (13) Human exposer to the COVID-19 in-house through flushing of the toilet and through defective plumbing systems, (14) Run-off water from rain water can accelerate the COVID-19 cases during major events and lastly (15) COVID-19 spreading through breaking of the WHO and countries' social distance rules, quarantine and lack of the proper hygiene. Proper hygiene, ethical following of the social distance and quarantine measures, mobile or on-site wastewater treatment plants on the affected areas with the presence of sufficient chlorination, ozonation or use of $\mathrm{UV}$ radiation to disinfect the water before discharge is an important aspect to eradicating the COVID19 in the water systems (Bhowmick et al. 2020). Sampling, analysis and interpretation of the data can assist to curb current and future health crisis. KWR Dutch carried out analysis via Dutch Municipal Health Services (GGD) as an early warning system. The increase of the COVID-19 RNA concentration in the wastewater system began with the first wave of the patient hospital admission and is expected to be tracked in the second wave. Figure 3 shows the concentration of the SAR-CoV-2 RNA in sewage water in Amsterdam, Netherland (March to Aug 2020) (Medema et al. 2020).

Where the red present the RNA genetic particles in the wastewater system, blue present the COVID-19 infected cases and grey present the number of patients in isolation and hospital. Currently, RT-qPCR assays is been developed for SARS-CoV-2 RNA specimen clinical testing and detection in the water system. Chlorine, UV radiation, ozone, chloramine is been used to inactivate and disinfect the water treatment system against the SARS-CoV-2. There is an urgent need for standard methodology/optimized standardized protocols for the detection and quantification of wastewater samples (Kitajima et al. 2020). 
Fig. 3 Concentration of the SAR-COV-2 RNA in sewage water at Amsterdam, Netherland (March to Aug 2020) (Medema et al. 2020)

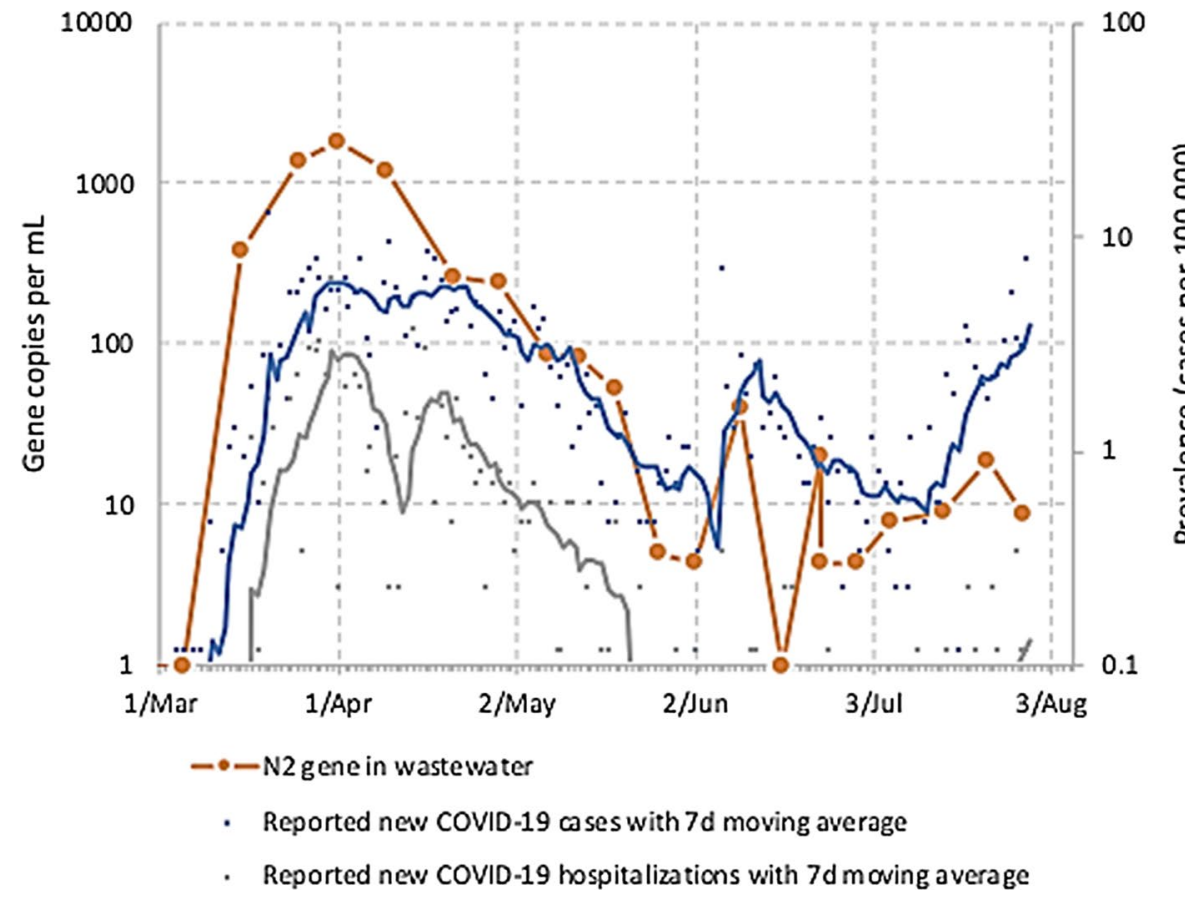

\section{Enveloped and non-enveloped pathogens in the water system}

Wastewater treatment plants are designed to reduce inactive pathogens such as viruses, protozoa, and bacteria that are harmful to human beings. Inadequate and inefficient wastewater treatment plants pose a health hazard to the receiving waters, irrigation system (agriculture), recreation centres and source of the drinking water. Most viruses in the water system are non-enveloped and consist only of the nucleic acids (RNA or DNA) engulfed in a capsid protein. An enveloped virus has an outer membrane with lipids and protein, relatively fragile and no longer infective when destroyed. Envelop viruses are much sensitive to heat, acids, $\mathrm{pH}$, disinfectants and detergents, and thus not able to withstand the stomach acidity medium for a long period. Enveloped viruses are assumed inactive and absent in the wastewater system. Enveloped viruses are responsible for many highprofile pandemic outbreaks like Swine Flu, Spanish Flu, SARS-CoV, middle east respiratory syndrome (MERS$\mathrm{CoV}$ ), Ebola and currently, the COVID-19 pandemic. Virus exposure to the UV radiation in form of sunlight or optimum UV wavelength range of the disinfection of 100-290 nm followed by chlorination before discharge to the receiving environment assist with denaturing and elimination of the COVID-19 in the water system. Inadequate treatment is widely presented in the ageing infrastructure of wastewater treatment plants in developing countries. This raises concern about the potential of the SARS-CoV-2 transmission via the water systems. Figure 4 shows a virion structure of the SARS-CoV-2 (W.R.C. 2020).

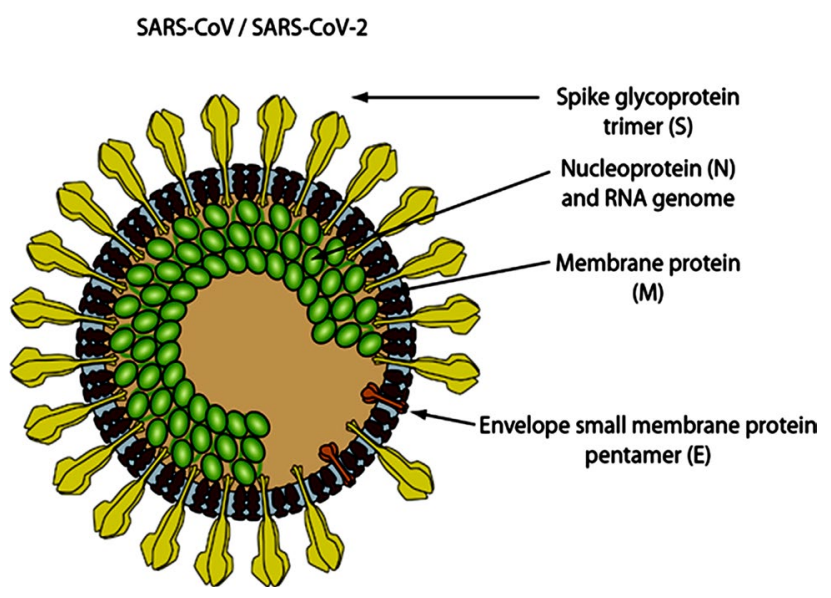

Fig. 4 Virion structure of the SARS-COV-2 (W.R.C. 2020)

The coronavirus is enveloped in a protein spherical structure with a diameter of $120 \mathrm{~nm}$. The envelope is a helical capsid that contains nucleoprotein and ribonucleic acid (RNA) genome. The 25 to $32 \mathrm{~kb}$ genome of COVID-19 is organized in two large frames (ORF1b and ORF1a) coding for the replicase polyprotein followed by terminal one-third of the genome with region encoding for the structure proteins (membrane, spike, envelope, and nucleocapsid protein) (La Rosa et al. 2020).

According to (La Rosa et al. 2020), COVID-19 was detected in hospital water, tap water and domestic sewage for 14 days at $4{ }^{\circ} \mathrm{C}$ and 2 days for $20^{\circ} \mathrm{C}$. The virus is persistent at low temperature and has rapid inactivation at high temperature that denature the proteins and the activity of 
the extra-enzymes. COVID-19 is reported to be persisted in urine for 17 days and stools for 3 days at $20^{\circ} \mathrm{C}$. Chlorination at $0.5 \mathrm{mg} / \mathrm{L}$ using chlorine and $2.19 \mathrm{mg} / \mathrm{L}$ using chlorine dioxide inactivate the SARS-CoV-2 in the wastewater system. It was reported to be inactive at $10 \mathrm{mg} / \mathrm{L}$ chlorine with a contact time of $10 \mathrm{~min}$ and $20 \mathrm{mg} / \mathrm{L}$ with a contact time of $1 \mathrm{~min}$ (La Rosa et al. 2020). Figure 5 shows the model used for the disinfection of the wastewater in the hospitals' based liquid chlorination, chlorine dioxide, ultra-violet, sodium hypochlorite technologies. The choice of the technology determines the economics of scale, safety precaution, operation management levels, critical skills, environment, investment and operation cost, and supply chain of the disinfectant (Wang et al. 2020).

The efficacy of the disinfectant techniques decreases in the order of ozone, chlorine dioxide to chlorine (Paleologos et al. 2020).

\section{Wastewater surveillance or wastewater-based epidemiology}

The economic limits on the medical screening for the SARS-CoV-2 or COVID-19 worldwide are turning to wastewater-based epidemiology (WBE) as great potential tools for assessing and management of the COVID-19 pandemic (Hart \& Halden 2020a). Current studies of the WBE or wastewater surveillance provide population-scale information for the detection of the hotspot, measure the scope of the outbreak, provide stakeholders with a decision platform, understand the impact of the demand in the health sector, track infection and measures, and provide an early warning for re-emergence of the pandemic outbreak. The emerging surveillance tool (WBE) employ modelling and computational analysis to examine the economy, feasibility,

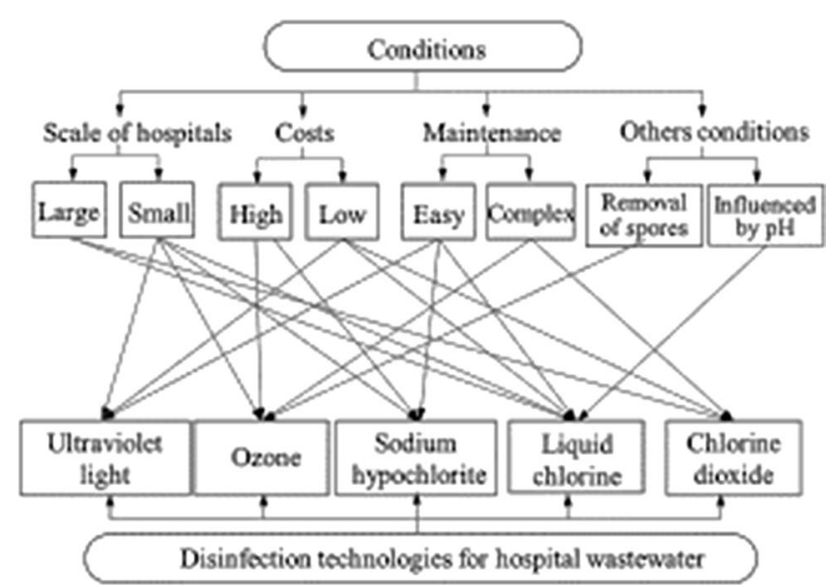

Fig. 5 Model for the disinfection of the wastewater from the hospital (Wang et al. 2020) opportunities, and challenges of active cases globally and locally based on the community wastewater of asymptomatic and symptomatic infected cases. The WBE surveillance of the large-scale population is cheap, rapid, with the potential robust tool of tracking COVID-19 than the clinical screening although it cannot replace the clinical screening. Significant cost savings are realized on the vulnerable population and enhance economic recovery with the containment of the COVID-19 in the community. Effective use of the WBE requires access to the wastewater system and mostly relies on integrated sampling and analysis for the decision-making process of global health surveillance. The WBE of the reverse transcription-polymerase chain reaction (RT-PCR) analysis is used to detect genetic materials in the RNA and is considered to be the most reliable means of the COVID19 diagnosis worldwide. Similar development of the use of the cartridges-based test is effective in the WBE purposes although with great challenges in the wastewater treatment plants. The cheapest and simplest testing technology is used of the paper-based rapid test kit to detect the potential of the COVID-19 carriers in the WWTPs. Cell culture is highly recommended for testing and analysis of the COVID-19 because of concerns related to biosafety (W.R.C. 2020). The important limitation of the WBE is the inability to identify the specific infected individual and their specific location (Hart \& Halden 2020a). The WBE aid in providing population-wide information and community health to the analysis of the antimicrobial resistance genes (ARGs) in the influent's wastewater and typically through the qPCR technology. The ethics in using WBE is low since no individual data is collected. However, privacy issues in a particular community should be accorded for potential stigmatisation. The key challenges that need to be tackled in the WBE surveillance of the water and wastewater concerning the COVID-19 are (i) non-existence biomarkers discovery pipeline for biological and chemical markers, (ii) account for the temporary population size fluctuations for an accurate estimate of the population, (iii) need for the biomarker extraction technology for the complex wastewater matrix, and lastly (iv) lack of the analytical tool that covers the sensitive, selective and multi-residual analysis and cost-effective over a wide range of the biomarker group (Sims \& Kasprzyk-Hordern 2020).

\section{Mathematical modelling of the COVID-19}

\section{SARS-COV-2 load into the wastewater treatment plant}

SARS-CoV-2 load into the wastewater treatment plant was estimated by (Zhang et al. 2020) using excretion rates in human stool by the assumption that the faecal load in the range of 100 to $400 \mathrm{~g}$ faecal per day per person and with the 
faecal specific density of the $1.06 \mathrm{~g} / \mathrm{ml}$. The estimation of the COVID-19 in wastewater can be calculated using Eq. 1 (Hart and Halden 2020a).

$N(t)=N_{o}\left(\frac{1}{2}\right)^{\frac{t}{t_{1}}}$

where $N(t)$ is the quantity remaining of the sample that did not decay after a time, $N_{o}$ is the initial excreted sample discharge into the collecting wastewater treatment system, $t$ is the time elapsed between excretion and sampling, $t_{1 / 2}$ is the half-life of the biomarker (Wilkinson, 2020).

\section{Mass balance in assessing the number of SAR-CoV-2 in wastewater}

The mass balance was used to estimate the prevalence of the SARS-CoV-2 infection on the total numbers of the RNA copies in wastewater per day by RT-qPCR. The number of the SARS-CoV-2 copies in the solid stool and on the infected person is calculated to determine the SARS-CoV-2 RNA in wastewater clinical samples (Ahmed et al. 2020):

Person infected with COVID - 19

$$
=\frac{\left(\frac{\text { RNA copies }}{\text { Litre of Waterwater }}\right) \times\left(\frac{\text { Litre of Wastewater }}{\text { Day }}\right)}{\left(\frac{\text { Faecal } g}{\text { Person - Day }}\right) \times\left(\frac{\text { RNA copies }}{\text { Faecal g }}\right)}
$$

The variability and uncertainty in the independent variables are incorporated using the Monte Carlos simulation model.

\section{Decay rate of the adjusted biomarkers}

Arrhenius equation was used to calculate the decay rate of the adjusted biomarker with the measured actual temperature of the wastewater as (Hart \& Halden 2020a; Pan et al. 2020):

$R_{2}=R_{1} \times Q_{10}^{\left(\left(T_{2}-T_{1}\right) / 10^{\circ} \mathrm{C}\right)}$

where $R$ is the initial rate of decay, $Q_{10}$ is the rate of change for each increment at the temperature of $10{ }^{\circ} \mathrm{C}$ and usually range from 2 to 3 . The half-life is calculated using Eq. 4 (Hart \& Halden 2020a):

$t_{\frac{1}{2}, 2}=t_{\frac{1}{2}, 1} \times \frac{\operatorname{In}(2)}{\operatorname{In}(2) \times Q_{10}^{\left(\left(T_{2}-T\right) / 10^{\circ} \mathrm{C}\right)}}$

where $t_{1 / 2,1}$ is half-life initial, $t_{1 / 2,2}$ is the half-life as spatially and seasonal adjustment to wastewater temperature, $T_{1}$ is the initial half-life temperature, $T_{2}$ is the calculated temperature and dependant on the rate change of the reaction (range from 2-3). Network analysis using ESRI ArcGIS is used to assign dry-weather flow loading based on population density and metering programme, and perform an accumulation analysis of wastewater flow from the households to the pipe network through the seasonally adjusted half-life in hours (Hart \& Halden 2020b).

\section{Mathematic modelling of spreading of the SAR-CoV-2 in a smart city}

The comprehensive model for the machine learning solution represents the transmission dynamics of the novel COVID-19 in a selected human population of the smart city from multiple dimensions. The assumption of the models is; there is contact tracing of all persons, some people are vaccinated, there is the existence of the government and personal quarantines, there is the existence of the government and personal treatment, the person-to-person contact is addressed, immunity can be lost at any time, the possibility of the re-infection of the recovery person, infection person can die through SAR-CoV-2, the infected person can die before being admitted in the hospital, an undetected infected person exists. The time-change of the population and infection rates are described by (Hashem et al. 2020):

The change of the population of the susceptible persons is presented as:

$\frac{\mathrm{d} s}{\mathrm{~d} t}=\wedge-\rho s-(1-\rho) S-\mu S+b_{2} G_{Q}+e S_{v}+b_{1} P_{Q}+\eta_{2} R$

The change of population of the vaccinated persons is presented as:

$\frac{\mathrm{d} S_{v}}{\mathrm{~d} t}=\rho s-\left(\alpha_{2}+e+\mu\right) S_{v}$

The change of population of the unvaccinated persons is presented as:

$\frac{\mathrm{d} S_{\mathrm{uv}}}{\mathrm{d} t}=(1-\rho) S-\left(\alpha_{2}+\mu\right) S_{\mathrm{uv}}$

The number of affected persons in contact with infected persons:

$\frac{\mathrm{d} E}{\mathrm{~d} t}=\alpha_{1} S_{\mathrm{uv}}+\alpha_{2} P S_{Q v}+\lambda S-\left(v_{1}+v_{2}+v_{3}+\mu\right) E$

The population of the person under authority (Government) quarantine:

$\frac{\mathrm{d} G_{Q}}{\mathrm{~d} t}=v_{1} E-\left(\mu+h_{1}+b_{2}\right) G_{Q}$

The population of the person under personal quarantine: 
$\frac{\mathrm{d} P_{Q}}{\mathrm{~d} t}=v_{3} E-\left(\mu+h_{2}+b_{1}\right) P_{Q}$

The population of infectious persons:

$\frac{\mathrm{d} l}{\mathrm{~d} t}=v_{2} E+h_{1} G_{Q}+h_{2} P_{Q}+\sigma-\left(f_{1}+f_{2}+\mu+\delta\right) l+\eta_{1} R$

The population of a person under personal treatment:

$\frac{\mathrm{d} P_{T}}{\mathrm{~d} t}=f_{1} l-\left(\tau_{2}+\mu+\delta_{2}\right) P_{T}$

The population of a person under personal treatment:

$\frac{\mathrm{d} G_{T}}{\mathrm{~d} t}=f_{2} l-\left(\tau_{1}+\mu+\delta_{1}\right) G_{T}$

The population of a person who recovered:

$\frac{\mathrm{d} R}{\mathrm{~d} t}=\tau_{2} P_{T}+\tau_{1} G_{T}-\left(\eta_{1}+\eta_{2}+\mu\right) R$

Figure 6 shows the summarized model of SAR-CoV-2 in a smart city.

Where $S$ is the individual susceptible, $S_{v}$ is the individuals susceptible that are vaccinated, $S_{\mathrm{UV}}$ is the individuals susceptible that are unvaccinated, $E$ is the individuals susceptible that are exposed and in contact with infected person, $G_{Q}$ is the exposed individual under Government quarantine,
$P_{Q}$ is the exposed individual under self-quarantine, $l$ is the infected individual, $P_{T}$ is the infected person under personal treatment, $G_{T}$ is the infected person under Government treatment, $R$ is the individuals that have recovered, ${ }^{\wedge}$ is the individual that join the susceptible population, $\rho$ is the rate of the susceptible vaccination, $\alpha_{l}$ is the rate at which the unvaccinated individual become infected when in contact with infected person, $\alpha_{1}$ is the rate at which the vaccinated individual become infected when in contact with infected person, $v_{l}$ is the rate at which Government quarantine individuals that contacted the virus, $\mathrm{v}_{2}$ rate at which individual become infected after been exposed, $v_{3}$ is the rate at which individual under personal quarantine that contacted the virus, $e$ is the rate at which vaccinated person losses immunity, $h_{1}$ is the rate at which persons under Government quarantine get infected, $h_{2}$ is the rate at which persons under self-quarantine get infected, $\lambda$ is the rate at which susceptible persons contact virus, $\sigma$ is the rate at which individual contact the virus through inhalation, $f_{l}$ is the rate at which infected person goes for the personal treatment, $f_{2}$ is the rate at which infected person goes for the Government treatment, $\tau_{1}$ is the rate individual treated by the Government recover, $\tau_{2}$ is the rate individual under self-treatment recover, $\delta_{1}$ is the rate of the COVID-19 related death under Government treatment, $\delta_{2}$ is the rate of the COVID-19 related death under self-treatment, $\mu$ is the rate of the natural death, ${ }_{\eta 1}$ rate of the second infection after recovery and ${ }_{\eta_{2}}$ is the rate at which
Fig. 6 A summarized model of the SAR-CoV-2 in a smart city (Hashem et al. 2020)

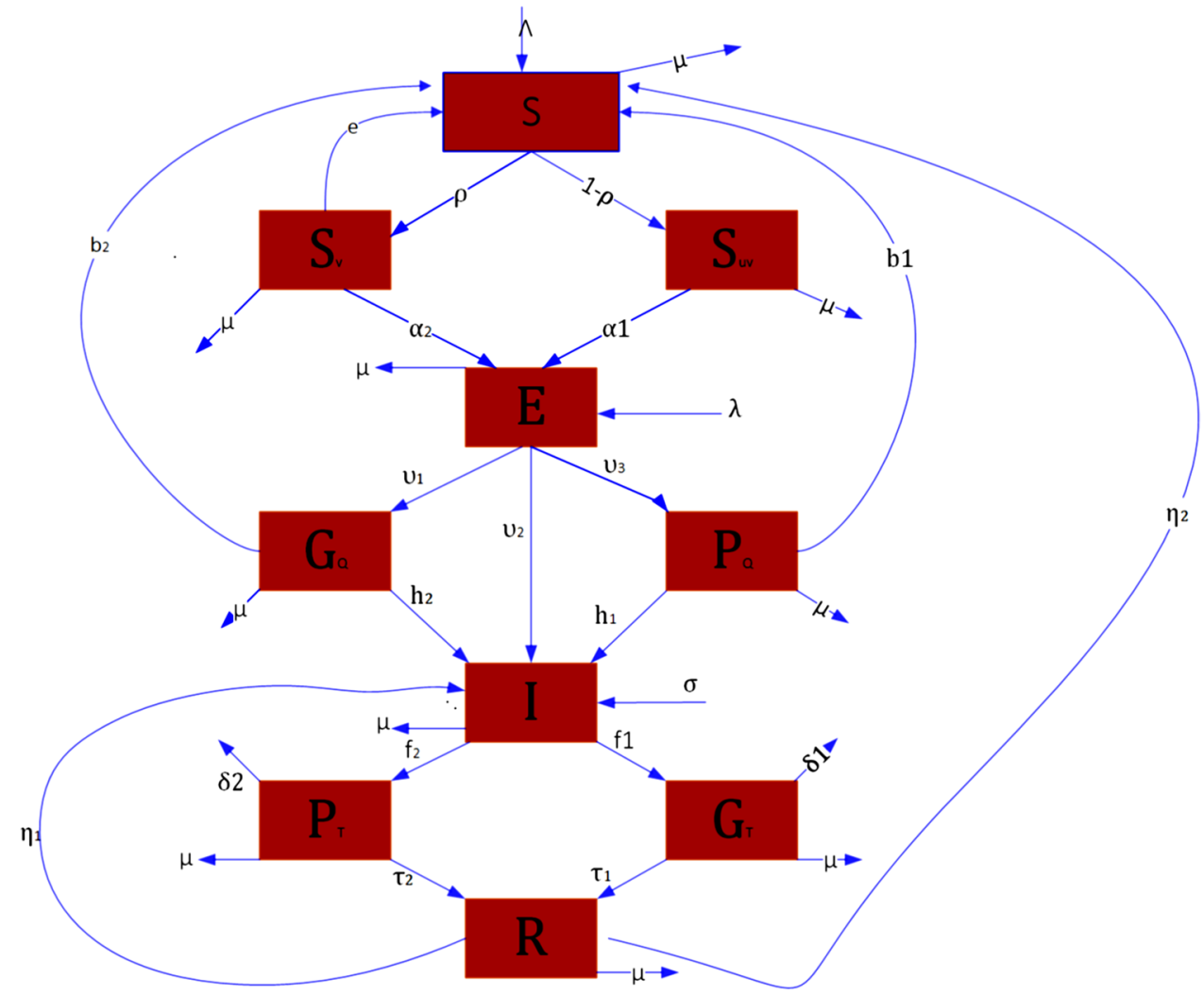


individual recover and become susceptible (Hashem et al. 2020).

\section{Prediction of the COVID-19 using ARIMA model}

Automatic regression integrated moving average (ARIMA) models are widely used for the prediction and forecasting of the epidemic syndrome and are significant in the COVID-19 outbreak. During the COVID-19 pandemic, ARIMA is useful in predicting the prevalence of COVID-19, understanding the trend of the outbreak and the epidemiological state of the particular region. They are instrumental in modelling temporary dependency structures in time series and easily explainable to the end-users during decision making. The ARIMA model is presented in Eq. 15 (Ceylan 2020).

$$
\begin{aligned}
Y_{t}= & \alpha+\phi_{1} Y_{t-1}+\phi_{2} Y_{t-2}+\ldots+\phi_{p} Y_{t-p} \\
& +\varepsilon_{t}-\theta_{1} \varepsilon_{t-1}-\theta_{2} \varepsilon_{t-2}-\ldots-\theta_{q} \varepsilon_{t-q}
\end{aligned}
$$

where $Y_{t}$ is the observed cases at time $t, p$ is the order of autoregression, $q$ is the order of the moving average, $Y_{t-1}$ to $Y_{1-P}$ linear current value of time series, $\varepsilon_{t-1}$ to $\varepsilon_{t-q}$ is the previous residual series, $\varepsilon$ is the observation of the current shock at time $t, \theta$ and $\phi$ is the moving average and autoregressive parameters, respectively, and $\alpha$ is the constant.

\section{Quantification of the SAR-CoV-2 infectiousness using contact tracing}

Infectiousness $\left(\beta_{\tau}\right)$ of the SAR-CoV-2 describes using a mathematical function. Infectiousness may change with time $\tau$ (days). The decomposed is presented into four sections namely; asymptomatic transmission $\left(\mathrm{R}_{\mathrm{A}}\right)$, symptomatic transmission $\left(R_{\mathrm{S}}\right)$, pre-symptomatic transmission $\left(R_{\mathrm{P}}\right)$, environmental transmission $\left(R_{\mathrm{E}}\right)$ with the summation of the four as $R_{o}$. The area under the curves indicates the total number of infection cases (Ferretti et al., 2020):

$$
\begin{aligned}
\beta_{\tau}=\underbrace{P_{a} x_{a} \beta_{s}(\tau)}_{\text {Asmptomatic }}+\underbrace{\left(1-P_{\mathrm{a}}\right)[1-s(\tau)] \beta_{s}(\tau)}_{\text {Pre - symptomatic }} \\
+\underbrace{\left(1-P_{\mathrm{a}}\right) s(\tau) \beta_{s}(\tau)}_{\text {Sympomatic }} \underbrace{\int_{l=0}^{\tau} \beta_{s}(\tau-1) E(l) \mathrm{d} l}_{\text {Environmental }}
\end{aligned}
$$

where $\beta_{s}(\tau)$ is the infectiousness of an individual of either pre-symptomatic or symptomatic at $\tau$ age of infection, $X_{a}$ is the relative infectiousness of the asymptomatic (central value 0.1$), P_{\mathrm{a}}$ is the proportional asymptomatic (central value 0.40$), s(\tau)$ is the incubation period of lognormal mean $\log$ (central value 1.64) and lognormal $\mathrm{d}_{\log }$ (central value $0.36), E(l)$ is the environmental infectiousness (central value $3)$.

\section{Cost analysis of the WBE and clinical screening}

The cost analysis of the WBE and clinical screening of an individual depends on the geospatial regions, safety requirements, labour cost, existing infrastructure. This is influenced by the relative cost savings that combine computed WBE and clinical screening as shown in Eqs. 17 and 18 (Hart \& Halden 2020a):

$\operatorname{Cost}_{p, \text { clinical }}=$ Cost $_{\text {reagents }} \times P$

$\operatorname{Cost}_{p, \text { wBE }}=\operatorname{Cost}_{\text {reagents }} \times N_{\text {wwtp }}$

where $\operatorname{Cost}_{p \text {, clinical }}$ is the cost of the total reagents need to test a population of a size $P, \operatorname{Cost}_{\mathrm{p}}$, wBE is the total cost of the reagent required in a community screening of the wastewater system of the population $P$ and the $N$ wastewater treatment plants. The key reagents of the WBE and clinical testing are real-time quantitative reverse transcription-polymerase chain reaction (QRT PCR) buffer and probes.

The performance of the predictive accuracy of the model selection can be validated using mean absolute error (MAE), root mean square error (RMSE) and mean absolute percentage error (MAPE).

\section{Digital revolution in combating COVID-19 in WASH}

The growth and advancement of the digital revolution (industry 4.0) including artificial intelligence, evolutional computational, data science, big data, quantum science, bioinformatics, nanotechnology, internet of thing (IoT), financial technology and blockchain create opportunities in service delivery of goods and services with better revenue and great opportunity in fighting the COVID-19 crisis. The change in water pattern has a direct impact on wastewater treatment and collection. The overall decline in water demand is leading to a decrease in water flow and an increase in wastewater parameters concentration with the potential of biosolid deposition. This would result in ineffective and inefficient treatment, development of odour and corrosion that damage the infrastructures. COVID-19 have raised demand on particular pharmaceutical products that contain high concentration and thus raise issues with the old age wastewater treatment plants. SARS-CoV-2 is shed in the faeces of the COVID-19 patients that is detectable in the wastewater treatment plants. Monitoring wastewater effluent (sewage epidemiology) can be a relevant source and tool of information the mitigate the spread of the virus (human health monitoring). Water equipped with a digital tool, sensors and big data analytics has a great potential to become an important source of information that provide real-time data 
for guideline and policy-making purposes. Figure 7 shows the water digital revolution encounter with COVID-19 Pandemic (Poch et al. 2020).

Water digitalization can provide techniques and tools that optimise the management and development of the water system. COVID-19 pandemic has revealed necessary measures to re-think and re-design the potential future of the pandemic outbreak. Water management and design of the water infrastructure require major changes to accommodate climate change, water cycle, reimaging of digitalization, infrastructure and privacy protection. The water digital revolution can accelerate with the consequence of the COVID-19 outbreak. The response is structured as (i) economic level by the contributor of the sustainable financing of the water utilities to support resource, asset, and energy management, (ii) technological level by enabling the optimal adjustment of utility operations and infrastructure. This is achieved by the use of the digital twin that integrates internet of thing (IoT) and big data analytics and lastly, (iii) water-human data level that increase the confidence of the general public and warn the decision and policy-makers of the signs of pandemics or general outbreak (Poch et al. 2020).

Machine learning is a field of science that depends on how computers learn from data using algorithms to identify knowledge from the real-world and improve performance.
It is embraced with artificial intelligence (AI) to model with self-learning ability that can combat COVID-19 in real-time. It correlates with pattern recognition, data mining, natural language processing, medical diagnosis, bioinformatics and video surveillance. Smart cities inter-connect urban society and data collection that brings out intrinsic and useful insights into the COVID-19 pandemic measures. Novel mathematical modelling is created to monitor the spread of the COVID-19 in the urban region. This is through detection, transmission, storage and analysis of the data using machine learning algorithms. This will assist in early detection of the COVID-19, prevent the spread, forecast and prediction of the pandemic, patient contact tracing, diagnosis and monitoring of the patients, patient monitoring, vaccine development and discovery, diagnose cases and provide a better understanding of the virus in the urban development (smart city/good city management). Figure 8 shows the framework for AI (machine learning and deep learning) in combating the COVID-19 pandemic (Hashem et al. 2020).

The smart city generated data are; (i) Statistical data that include daily statistics of identity, number of COVID-19 positive cases, number of birth and death, recovery cases, COVID-19 active cases; (ii) Epidemiological data contains clinical test data with different medication, drug trials, nonmeasurement for the disease tracking, protein structure
Fig. 7 Water digital revolution encounter with COVID-19 Pandemic (Poch et al. 2020)

\section{New Water-Human-Data Nexus}

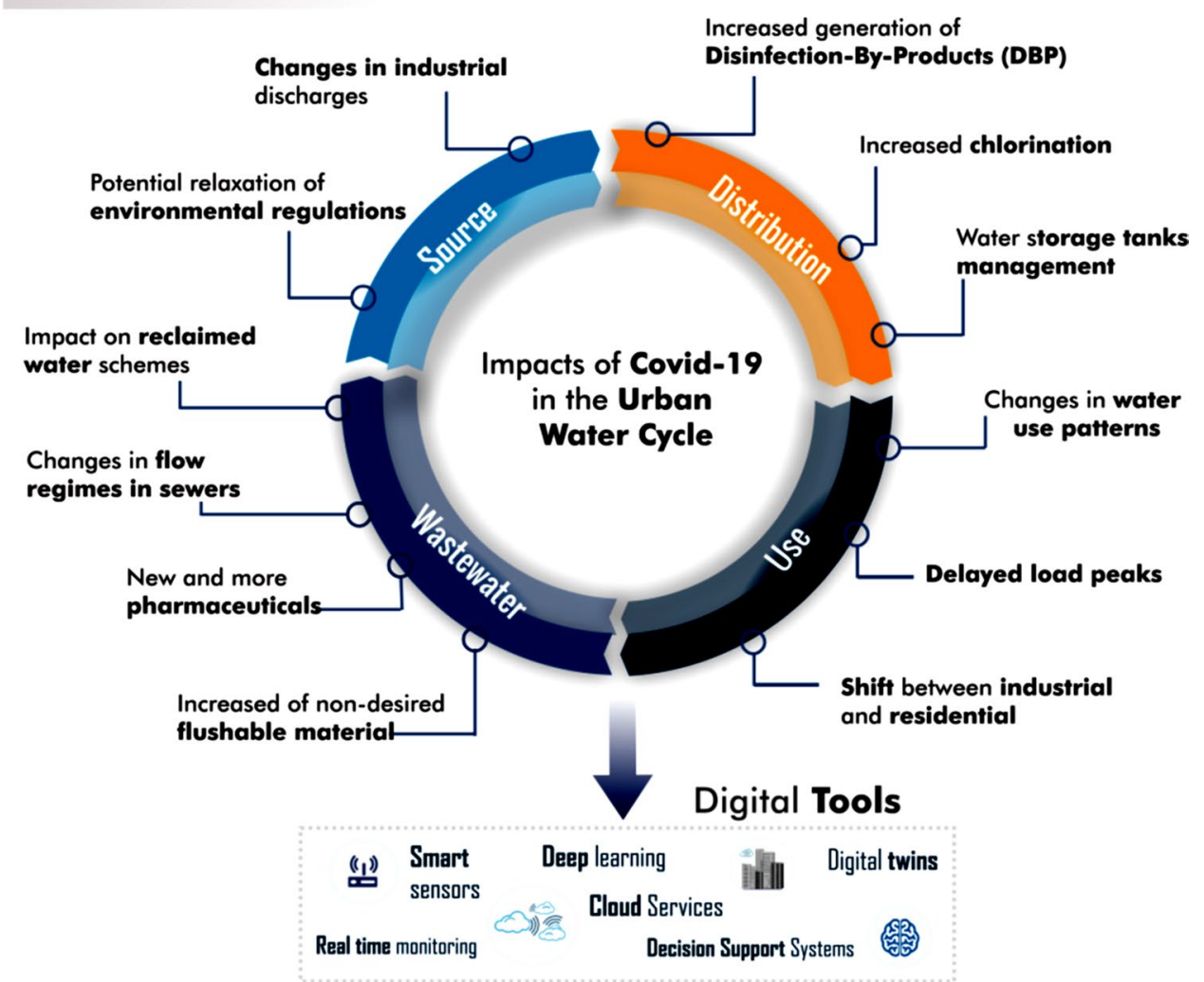


prediction, medical imaging for diagnosis, improving viral and testing patient history, response to the patient on medication and contact tracing; and (iii) Real-time surveillance generated from cameras (facial recognition) and sensors (e.g. temperature) can detect COVID-19 symptoms and assist prevent spreading of COVID-19 (Hashem et al. 2020).

\section{Predictive analytics of the COVID-19}

Predictive analytics using data techniques (statistical analysis, machine learning, deep learning and predictive models and algorithms) is a major pillar to predictive and decisionmaking tools in the COVID-19 pandemic. Prediction models using machine learning and computer vision are enforced for identifying most of the diseases using patient image patients. The machine learning approach of the COVID-19 prediction includes person infection, positively diagnosis, mortality rate, and contact tracing. Prediction mortality rate using machine learning algorithms (decision tree, random forest, support vector machines, logistic regression and artificial neural networks) is been effective. COVID-Net uses a convolutional neural network design for the identification of COVID-19 cases using chest X-ray (CXR) images. Modified susceptible exposed infections removed (SEIR) model and COVID-19 AI-prediction pandemics epidemiological data with population migration data can effectively predict the size and peaks of the COVID-19. The artificial intelligencebased automated computer tomography (CT) image analysis using deep learning to detect images, track and quantify and classify can identify patients with COVID-19. The same concept can be used in wastewater treatment to detect and track the COVID-19. Patients information-based algorithms
(PIBA) can be used to determine the COVID-19 patients mortality rate. Machine learning can learn from the protein and drug structure and predict and integration and response in the vaccine discovery, predict the signature of vaccine reactogenicity and immunogenicity. Blockchain technology can be applied in health passports (digital passports) to track the COVID-19 in travellers with the provision of encrypted patient records with high cyber-security (Hashem et al. 2020). The challenges of the data science and artificial intelligence (image analysis and processing, textual data mining, embedded sensing and audio analysis) in the COVID-19 pandemic are limitation of the data, security, ethics and privacy, need for multi-disciplinary collaboration, data beyond the border, data modalities and lack of critical skills in the fourth industrial revolution (4IR) (Alabool et al. 2020). Introduction of the cloud-assisted wastewater management system (CAWMS) with the IoT, sensors, the android application can be able to handle the wastewater big data and avail them in real-time (Chellaswamy et al, 2019).

\section{Integration of the smart water system with digital tools}

Exploration of the ICT in high-fidelity visualisation data and human interpretation is a powerful approach for conveying potential hypotheses and exploring correlations. The use of water-smart tools like self-organising maps (SOM) equipped with data science of unsupervised artificial neural networks (ANNs) is demonstrated in the water distribution system (WDS), data mining for microbial, and physio-chemical data in the wastewater treatment plants, clustering of the water quality, asset management, water leakage, hydraulic

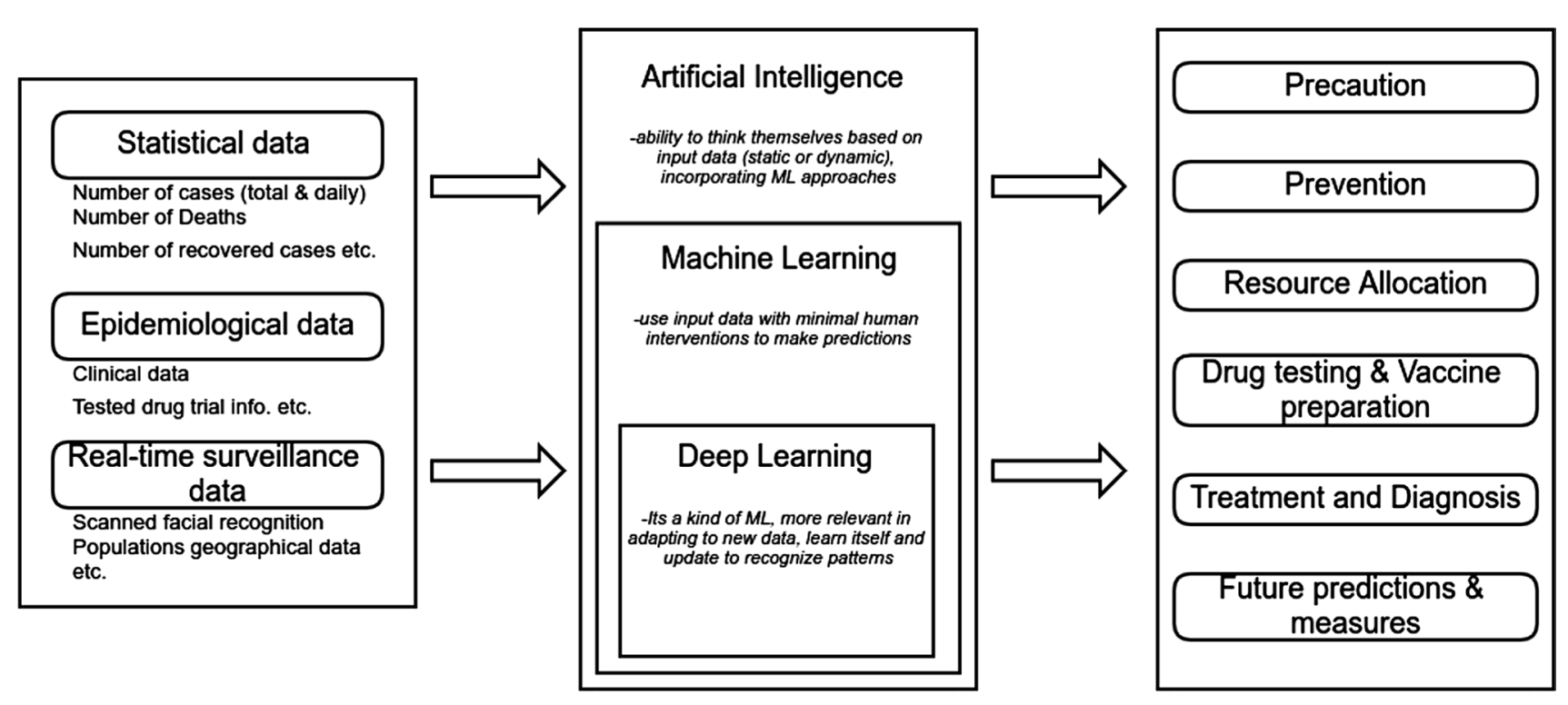

Fig. 8 Application of artificial intelligence (machine and deep learning) in combatting COVID-19 (Hashem et al. 2020) 
modelling and geospatial behaviour. Some of the hydroinformatic tools like mathematical modelling, computational intelligence, data science (i.e. big data, deep/machine learning, cloud computing, blockchain, IoT, smart network, knowledge discovery) provides decision-making system that of a major solution to the smart water network. Figure 9 shows the integration of the smart water system with water digitalization tools (Mounce 2020).

The intelligent monitoring system consists of (i) supervisory control and data acquisition (SCADA) system for data source equipped with programmable logical controller (PLC) and ole for process control (OPC) servers for data distribution services and communication, (ii) configure and design distribution of the data distribution services used to transmit real-time data and software data monitoring and transfer, (iii) variable selection based on historical and input variables that are dependants on the principal component analysis (PCA) algorithms for training and testing and lastly (iv) integrated online data-driven soft sensor, data acquisition and control system (DAC) with adjusted parameters by utilization of the historical data to train a fuzzy neural network (FNN) and adaptive learning rate used for the initialization of the real monitoring models. Figure 10 shows the water intelligence monitoring system (Han et al. 2018).

\section{Digital twins in disruption of the water and wastewater treatment technology}

The introduction of the SCADA accelerated the water and wastewater utility systems. The on-set of the next generation flight simulator (digital twins) enables dynamic process simulation, staff training, reduce risks, optimized operations, visual integration designs, pumping stations, flow operation, SCADA screens, instrumentation and piping network with a connection of the internet of things (IoT). Digital twin in smart water and wastewater management is defined as virtual replica system that embodies and simulate multi-physical components, multi-scale in the real system of the wastewater, water, stormwater, rivers with real-time sensors, historical performance data and deep/machine learning (AI). It has access to the current plant and can predict the future integration of the parameters in the plant with intelligent functions of model-based systems (Kolditz et al. 2019). Digital twins form a central repository for information and analysis of the treatment plant through the use of the operator training, dynamic process modelling, event of the database of flow, predictive analytics, hydraulic modelling with calibrated data, optimization, machine learning and data analytics and lastly reduce risk with the improvement of the design and operations of the systems. In the COVID-19 era, digital twin in a decentralized water and wastewater treatment can simulate the control, hydraulic and water quality with the provision of the full motion pictures, the interaction of a facility before building. Calibration of the models enables effective control systems (using IoT) and sensors that are more robust to dynamic process and data analytics while ensuring high quality treated water for reuse (Curl et al. 2019). Figure 11 shows the approach to water and wastewater management using a digital twin of the physical replica system. The performance optimization of the plant can be described based on the reactive data analysis

Fig. 9 Integration of the smart water system with water digitalization tools (Mounce 2020)

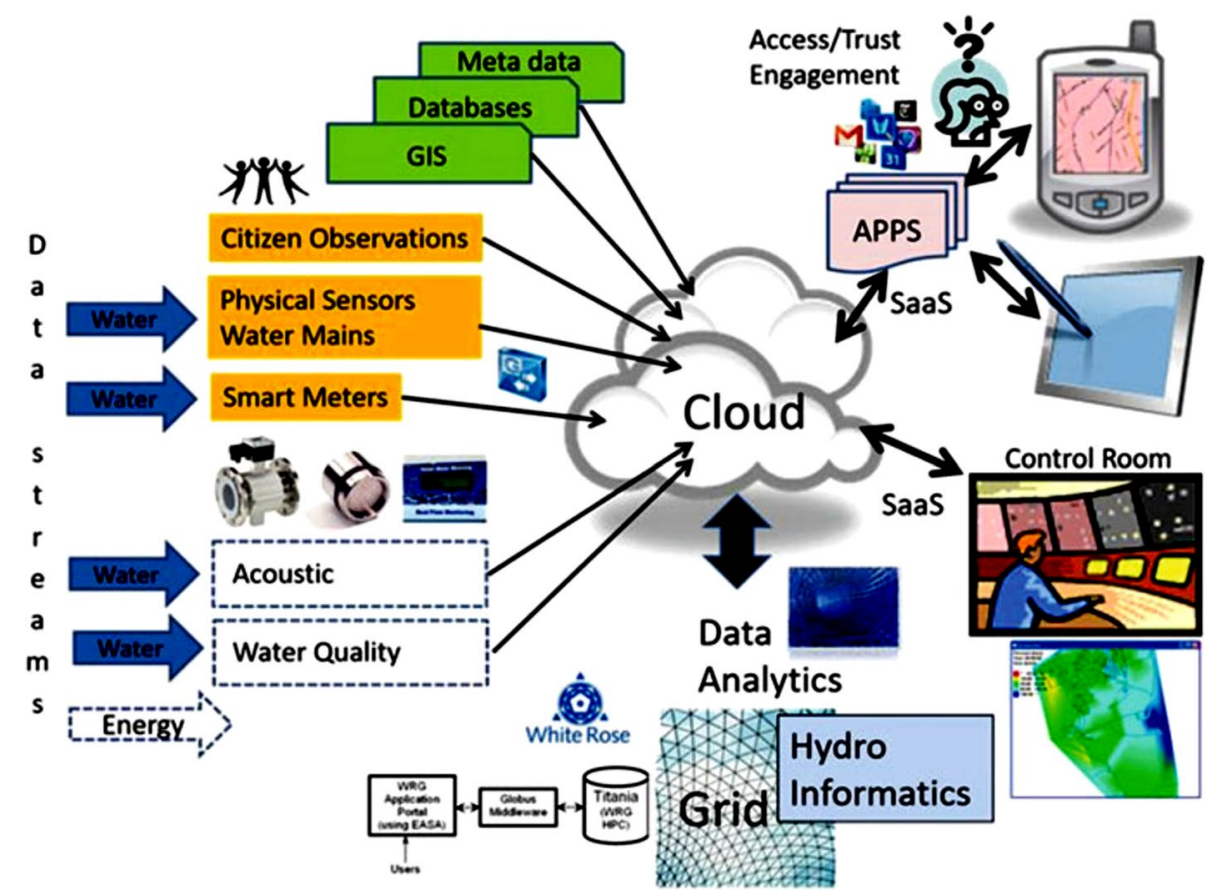




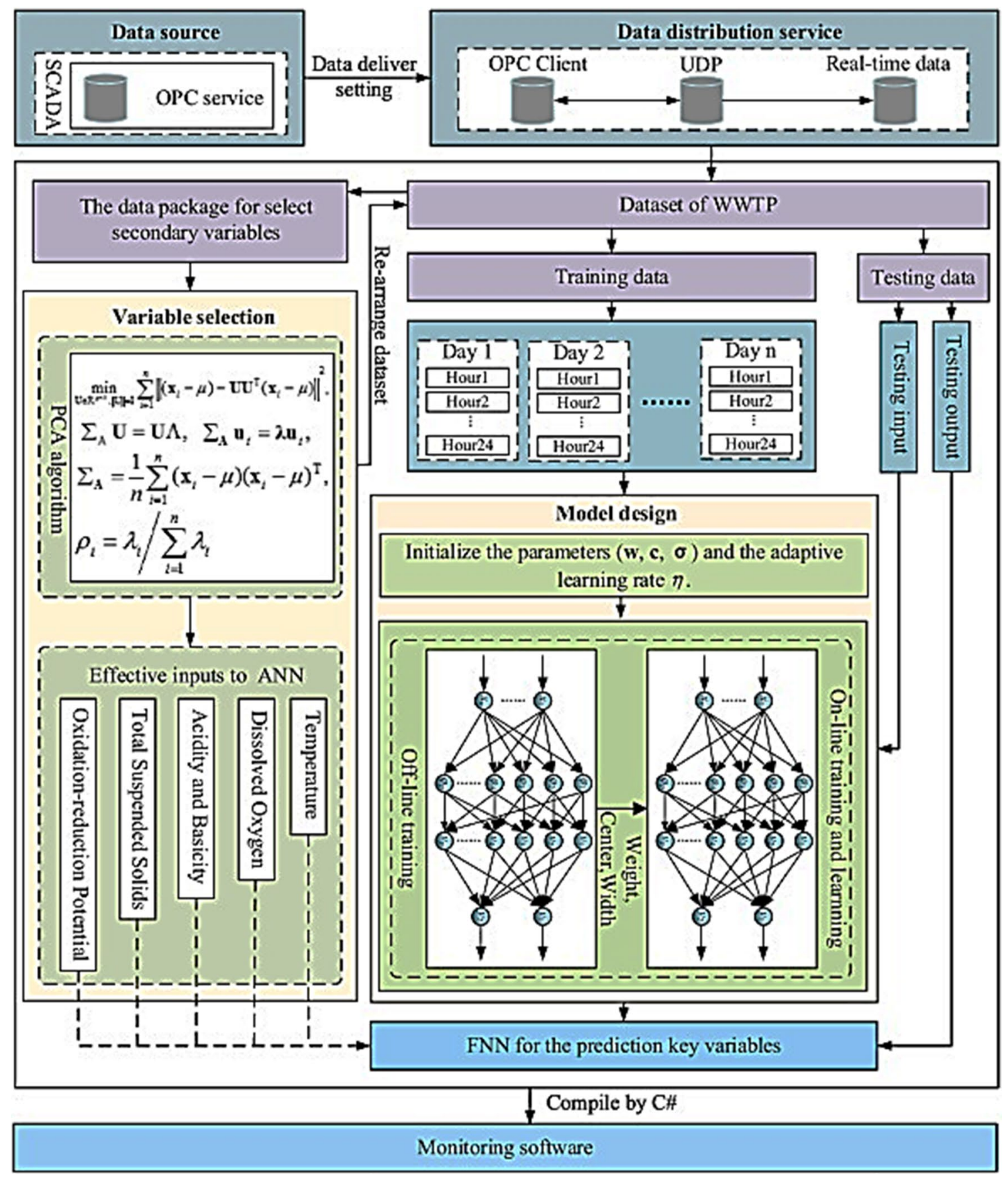

Fig. 10 Water intelligence monitoring system (Han et al. 2018)

that is provided by the sensors under pre-defined operation at real-time control (RTC) and monitoring. Better optimization of the plant leads to the increased removal of the current pollutants and emerging pollutants, reduce energy and chemical consumption, increase capacity and early detection of the emerging pollutant (DHI 2020).

The urban water cycle (UWC) based on the urban drainage system (UDS) and water supply systems (WSS) equipped with cyber-physical systems (CPS) provides disruptive technology for efficient and critical systems. This exploits the framework of the supervisor and control to the computational and control system of the physical structure, scheduling through strategic computation using PLC, digital twin, of the digital presentation of the physical plant to obtain hydraulic or quality variables (provide extra soft-sensor data) by model predictive control (MPC) with virtual simulation tools for collection system (i.e. Infoworks and SWMM), for drinking water 


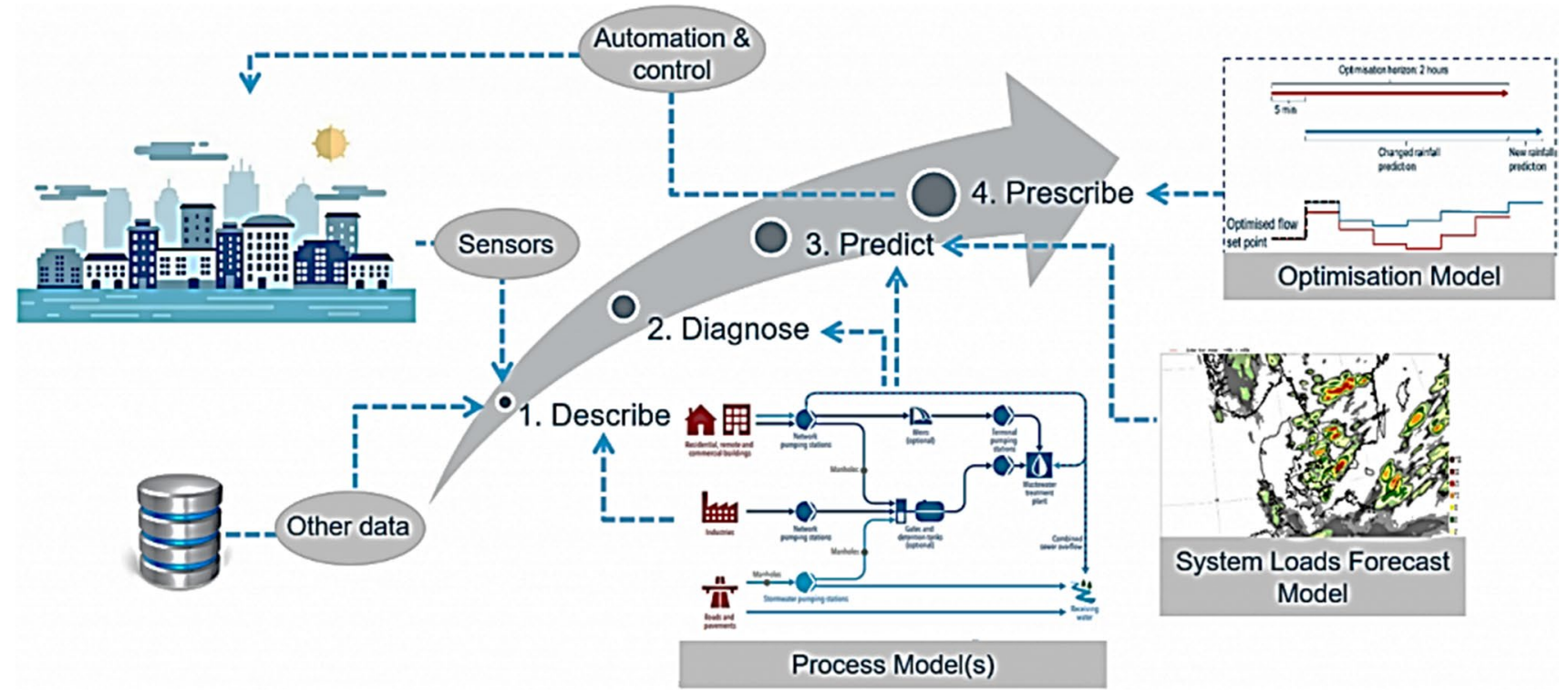

Fig. 11 Approach to the water and wastewater management using the digital twin of the physical replica system (DHI 2020)

distribution network (i.e. EPANET) for water resource recovery facilities WRRFs (i.e. Biowin, Simba, Sumo, GPS-X), groundwater and water-related domains (i.e. Simulink-MATLAB, DHI) evaluation of control, performance and desired accuracy and lastly water users and environment layers that receive the impact of the control action in the CPS. Figure 12 shows the wastewater multilayer cyber-physical system (CPS) based on the supervised structure (Ferretti et al. 2020; Garrido-Baserba et al. 2020).

\section{Geospatial and spatial-statistical analysis of the COVID-19 pandemic}

Geographical information system (GIS) is a valuable tool for social mobilizations and community response that minimizes the regularity of sacrificing the unprotected marginalized population. Understanding the spatial-temporal dynamics of COVID-19 is critical to its mitigation. The diversity of the disease mapping is categorized in health and social geography, spatial temporally analysis data mining, environmental variables and web-based mapping. Health geography
Fig. 12 Wastewater multi-layer cyber-physical system (CPS) based on the supervised structure (Ferretti et al. 2020)
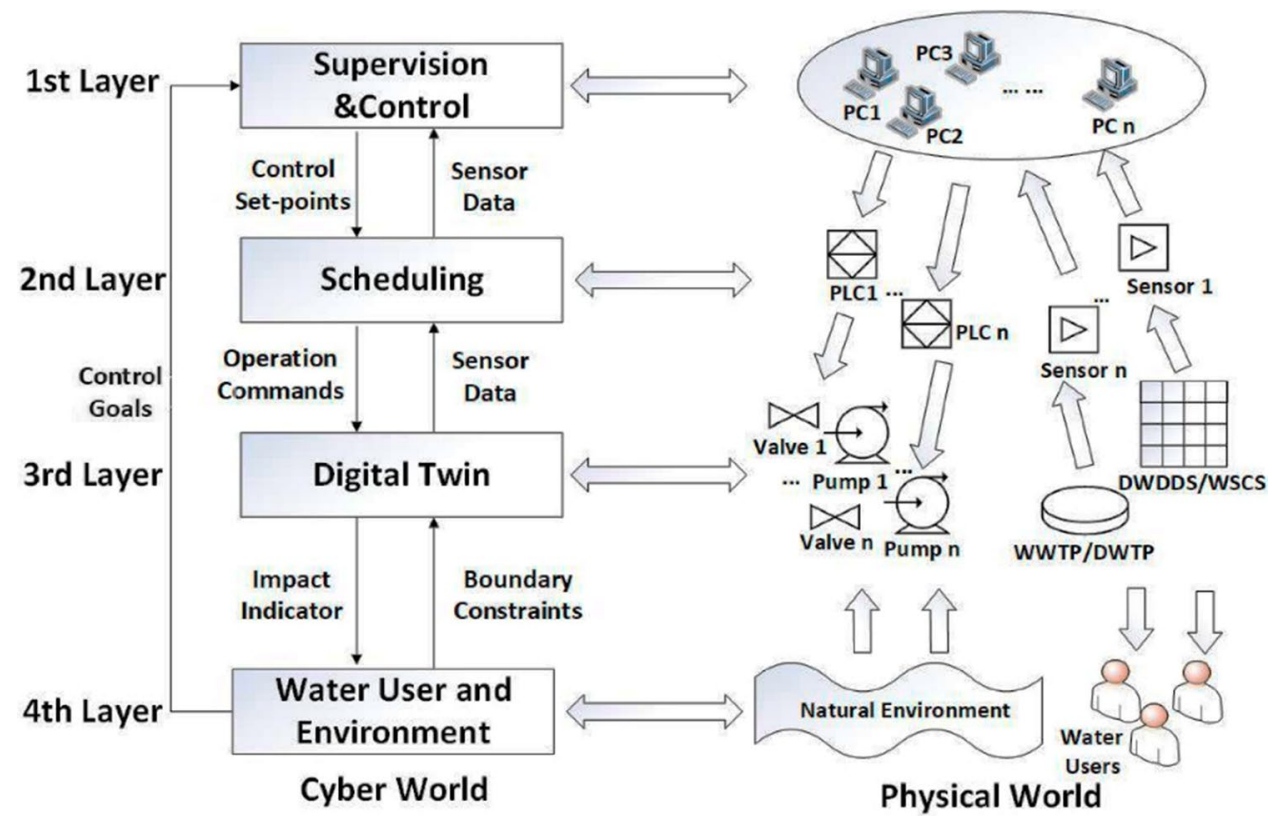
highlights the interaction of affected actors, public health and first respondent to estimate the disease propagation and new outbreaks. Spatial-temporal helps clarify the extent of the COVID-19 and the impact of the pandemic on the planners, policy and decision-makers and community. Using geospatial big data (i.e. human mobility patterns from mobile phones) can quantify a scale of an event that facilitates appropriate policy making such as the COVID-19 pandemic. Web-based view and mapping have a very important role in the provision and dissemination of information of the COVID-19. They are effective for the pandemic spatial representation and evolution of non-specialized and specialized internet users. Interdisciplinary correlation is required on health policy, health services and controls measures, formulation of appropriate political and scientific response, mapping and tracking of the human movements. To date, spatial-temporal analysis and disease mapping, environmental variables, social and health geography, data mining and web-based mapping have been the recurrent topic in the COVID-19 pandemic (Franch-Pardo et al. 2020).

\section{Biosensors and nanoscale tools for COVID-19 pandemics}

WASH practitioners and public health stakeholders should adopt bio-sensors as a smart sanitation technology into their infrastructure and sanitation products to mitigate, detect and monitor the emerging contaminants that have global health potential and community level (disease surveillance) and personal level (for diagnostics) (Rary et al. 2020). The development of disruptive and innovative bio-sensor-based nanostructured materials is promising for the detection of waterborne pathogens in water samples (Bilal et al. 2020). The development of the biosensor for prognosis and early detection of the COVID-19 strains should address the features (Bhalla et al. 2020):

(i) High selectivity and ability to exclusively detect the analytes in the presence of other contaminants.

(ii) Highly sensitive to affinity, spacing and specificity of the target probes in self-assembled mono-layers or biorecognition elements on the sensor surface. For the COVID-19, the sensor should be able to detect biomarkers at a low concentration and produce easily readable output. The specificity and sensitivity of the biosensor towards the early detection of the COVID-19 are the key aspect in the innovation and disruption of the bio-sensors.

(iii) Quick response time of the diagnosis tool should be rapid and of paramount importance.

(iv) Multiplexity system should be allowed to detect the multiple biomarkers of the COVID-19 with accurate, quick and early detection of the disease. This can be achieved with physical isolation of different areas of the sensor surface.

(v) A trade-off of the multi-mode sensors and increasing more than one mode of sensing and physical dimension and computational time of sensing could provide crossvalidation of the sensing results with accuracy.

(vi) To avoid contamination, a single-use (disposable sensors system) of the sensors is needed to avoid contamination and sensing system. Integrating the sensors with the mobile app provide advantages of cost-effectiveness and connectivity and periodic data of the centralized health systems.

(vii) The sensors electrodes should be easy to use and this ensures that a large number of testing kits are produced for self-testing and preparedness of the self-isolation.

(viii) It is effective to have low-cost bio-sensors that communities can afford and easily available at shops selves i.e. disposable electrodes such as paper-based and screen-printed electrodes. Other biosensors are electronic sensor (field-effect-transistors-(FET), electrochemical detection using carbon electrodes), optical biosensors using principles of plasmonic such as surface plasmon sensors (SPR), plasmonic photothermal (PPT), localised surface plasmon resonance (LSPR), nanoscale direct visualization, characterization and diagnosis of the virus using electron microscopy (EM), $\mathrm{X}$-ray diffraction (XRD) and atomic force microscopy (AFM).

(ix) The COVID-19 have raised the manufacturing demand of the sensors and thus this call for low cost, high available sensors that cover the total population and geographical areas. The advancement of 3D printing and machine moulding can help increase production levels.

(x) Compatibility of a biosensor to the central health systems is of importance along with the autonomy measurement that collects information in real-time. Improved networked health care system with the mobile application can provide health care and Government policy-makers with real-time accurate information and provide a framework of containment and mitigation of the COVID-19.

\section{Digital contact tracing for COVID-19 using a mobile apps}

Contact tracing using the mobile apps in a novel COVID19 pandemic is a vital tool in modern society. The tools assist to track the infected patients and identifying their close contacts. There still exists a huge gap in cybersecurity, privacy and ethics when using the tracking app and tracking wastewater surveillance from the source. 
Publishing contacts of the infected patients by the Government attract snoopers to track and identify the patient locations. This gives no reasonable privacy-conscious society where the app simply broadcasts phone numbers and names with phones log information. The most reasonable system uses the unique user-ID for each individual through blockchain technology to broadcast information. Another mechanism used by Singapore is the use of the broadcast random time-varying tokens as temporary IDs. App administration (Government and private agency) privacy and protection of the users are of great challenge as well. The absence of a decentralized peer-to-peer system on information systems among apps and phones needs protective measures based on cryptography and coordinated servers. The use of placing Bluetooth receivers (proximity network) in public places needs to be improved with the use of the GPS location for contact mapping of the infected patients. Bluetooth low energy technology can only track over a short distance (few metres). The hybrid system of GPS, cryptographic app and Bluetooth low energy proximity network with temporary generated ID and PushToken proves useful to public health decision-makers in modelling disease using contact tracing. The list of logged IDs are encrypted and stored locally on user phones. Using secure log and user location history resolved with appropriate redactions and safeguards as used in Singapore and Europe. South Korea trading off of the privacy enhance the privacy of the individual who is trying to see if they are exposed. The use of the cryptographic protocols with app-based contact tracing (periodic encrypts) assist in tracing the patients without compromising the privacy and ethics of the patients and thus a privacy-friendly tracing approach. Figure 13 shows COVID-19 contact tracing using a mobile app (IOS and Android) with consideration of the privacy and trade-off (Abeler et al. 2020; Cho et al. 2020; Ferretti et al., 2020).

The question to the equality, privacy protection and fairness the digital COVID-19 contact tracing needs an ethical digital intervention that comes with a price. COVID-19 may be temporary but the risk is permanent with vulnerable health people records, social interaction and movement. The four ethical interventions and principles are necessity, proportionality, scientifically valid and time-bound during the COVID-19 pandemic. Developers, Government agencies, and deployers of the COVID-19 contact tracing app should address the ethical hypothesis before deploying it to the public (Morley et al. 2020; Parker et al. 2020).

\section{COVID-19 with robotics}

The design and implementation of the robot with the control system on the grouping of the fuzzy logic control (FLC) with sliding mode control and validation in real-time digital simulator Opal-RT give a high degree of robustness in robotics technology. The use of robots' systems has been used for the social distancing of the patient and medical personnel's

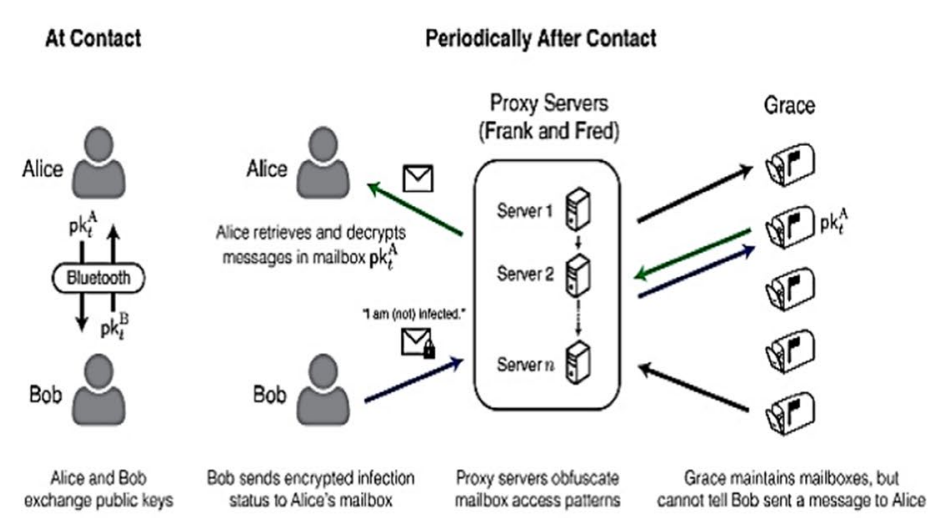

How the app would track coronavirus contacts

$\int$ Has Covid-19, but is unaware as has no symptoms
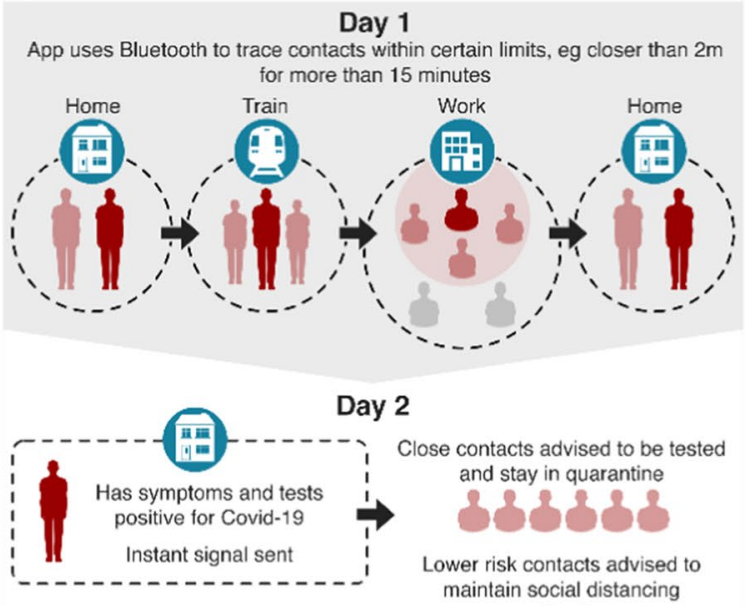

maintain social distancing

Decontaminated

요요용

Fig. 13 COVID-19 contact tracing using a mobile app (IOS and Android) with consideration of the privacy and trade-off (Abeler et al. 2020; Cho et al. 2020; Ferretti et al. 2020) 
while providing all the services required by the patient. This assist reduces the risk of spreading COVID-19. Other robots in the COVID-19 includes the sanitizing drone robots, delivery drone robots, speci-minder robot for efficient specimen delivery systems, robotic drugs, chatbot, fintech, hospital blood sample courier robot, helpmate robots, automated guided vehicles. Figure 14 shows the control methodologies and mathematical modelling of the tracking trajectory robot for maintaining social distance (Qureshi et al. 2020).

\section{COVID-19 knowledge management and building information modelling}

COVID-19 is a wake-up call cross-boundary and cross-sectoral. Water is identified as a key risk to our society from the potential source to end-use. It is of importance to the waterhealth-digital nexus. The resource availability and contribution to creating green jobs and sustainable growth provide resilience and opportunities in infrastructure investment, climate change, digitalization and smart-water society, waterdriven sustainable growth and emerging pollutant monitoring, biodiversity, food resilience, sustainable tourism, circular economy through reuse of water, integrated water efficiency, water cyber-security and cyber terrorism, water risk management, transport, and trade of energy. The emerging disruption of building information modelling (BIM) and knowledge management (KM) presents an opportunity for the water industry during the COVID-19 and post COVID19. This enhances a real-time tracking and response of the COVID-19 through the industry supply chain (Suresh et al. 2019). The WHO guidelines of COVID-19 on wastewater system is investing in the wastewater management strategy through economic valuation, working in partnership, providing a sustainable solution, investment on the decentralized WWTP, share knowledge and raising awareness on the key challenges with stakeholders, digitalization of the water system, proper monitoring and reporting, efficacy treatment of the wastewater, effective reuse of treated water, improvement of the working conditions, automation and control of the wastewater treatment plants (U.N.E.P 2020).

\section{Conclusion}

The major concern underlying the potential burden to the COVID-19 in the low- and middle-income countries (LMICs) are vulnerability due to epidemiological, high demand for the health system and availability of the intensive care, control measures of the social protection and economic package. The WBE surveillance of the large-scale population is cheap, rapid, with the potential robust tool of tracking COVID-19 than the clinical screening although it cannot replace the clinical screening. COVID-19 pandemic has highlighted the importance of proper management of water, sanitation and hygiene (WASH) and protection of human health. Contaminated wastewater with pathogens and exposure to human beings and animals play a major role in the spread of the diseases. Surveillance and tracking of the pathogens in the wastewater are key to the early warning system (EWS) and public health strategy monitoring of the COVID-19. Proper hygiene, ethical following of the social distance and quarantine measures, mobile or onsite wastewater treatment plants on the affected areas with the presence of sufficient chlorination, ozonation or use of $\mathrm{UV}$ radiation to disinfect the water before discharge is an important aspect to eradicating the COVID-19 in the water systems. Currently, RT-qPCR assays is been developed for SARS-CoV-2 RNA specimen clinical testing and detection in the water system. Chlorine, UV radiation, ozone, chloramine is been used to inactivate and disinfect the water treatment system against the SARS-CoV-2. Chlorination at $0.5 \mathrm{mg} / \mathrm{L}$ using chlorine and $2.19 \mathrm{mg} / \mathrm{L}$ using chlorine dioxide inactivate the SARS-CoV-2 in the wastewater system. It was reported to be inactive at $10 \mathrm{mg} / \mathrm{L}$ chlorine with the
Fig. 14 Control methodologies and mathematical modelling of the tracking trajectory robot for maintaining social distance (Qureshi et al. 2020)

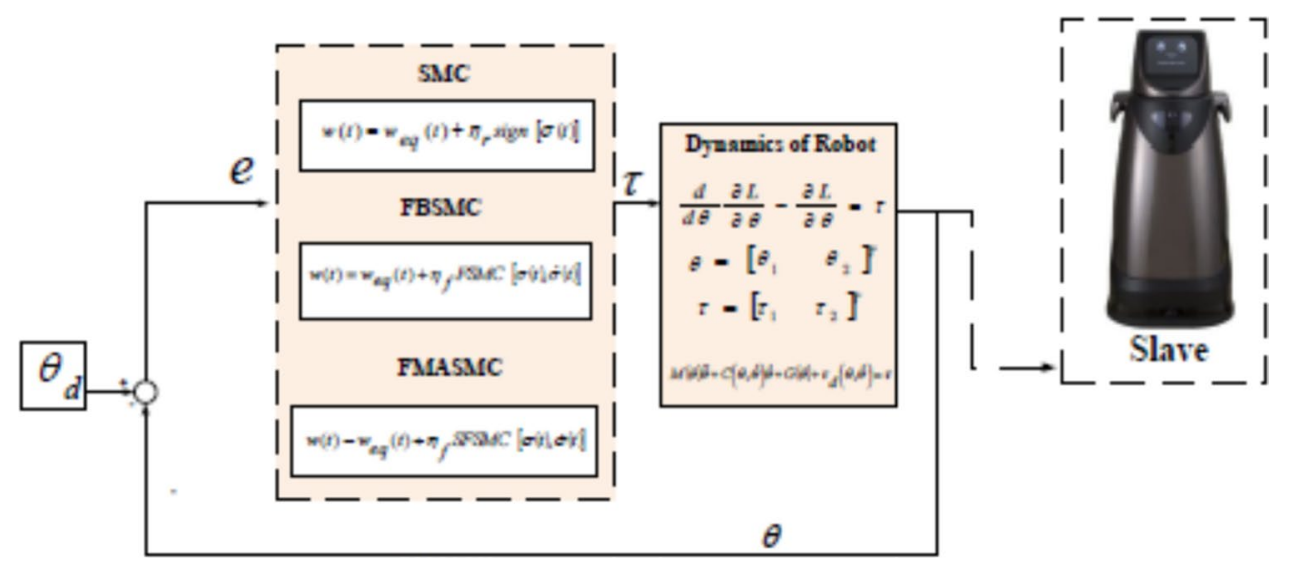


contact time of $10 \mathrm{~min}$ and $20 \mathrm{mg} / \mathrm{L}$ with the contact time of $1 \mathrm{~min}$, respectively. The emerging surveillance tool (WBE) employ modelling and computational analysis to examine the economy, feasibility, opportunities, and challenges of active cases globally and locally based on the community wastewater of asymptomatic and symptomatic infected cases. The WBE surveillance of the large-scale population is cheap, rapid, with the potential robust tool of tracking COVID-19 than the clinical screening although it cannot replace the clinical screening. The ethics in using WBE is low since no individual data is collected.

The growth and advancement of the digital revolution (industry 4.0) including artificial intelligence, evolutional computational, data science, big data, quantum science, bioinformatics, nanotechnology, internet of thing (IoT), financial technology and blockchain create opportunities in services delivery of good and services with better revenue and great opportunity in fighting the COVID-19 crisis. Sustainable water management and design of the water infrastructure that addresses social-economic, health, environmental and technical aspects require major changes that accommodate climate change, water cycle, digitalization (intelligent system) and privacy protection. The water digital revolution can accelerate with the consequence of the COVID-19 outbreak. Predictive analytics using data techniques (statistical analysis, machine learning, deep learning and predictive models and algorithms) is a major pillar to predictive and decision-making tools in the COVID-19 pandemic. In the COVID-19 era, digital twin in a decentralized water and wastewater treatment can simulate the control, hydraulic and water quality with a provision of the full motion pictures and the interaction of a facility before design, construction and monitoring. Spatial-temporal helps clarify the extent of the COVID-19 and the impact of the pandemic on the planners, policy and decision-makers and community. WASH practitioners and public health stakeholders should adopt bio-sensors as a smart sanitation technology into their infrastructure and sanitation products to mitigate, detect and monitor the emerging contaminants that have global health potential and community level (disease surveillance) and personal level (for diagnostics). Contact tracing using a mobile app in a novel COVID-19 pandemic is a vital tool in modern society. There still exists a huge gap in cyber-security, privacy and ethics when using the tracking app and tracking wastewater surveillance from the source. COVID-19 is a wake-up call cross-boundary and cross-sectoral. It is of importance to the water-health nexus.

Acknowledgements The authors wish to acknowledge the University of Johannesburg (UJ), Water Research Commission (WRC), Afrika Kommt! (GIZ and SAP SE), UJ-PEETS, UNEP, Esther Nabadda, Mary Matheri, Loise Wangaru, Gladtech International, WISA for capacity building and knowledge transfer.
Authors contribution All authors contributed to the study conception and design. Material preparation and analysis were performed by Dr ANM, Prof MB, Dr CNK, Prof JCN. The first draft of the manuscript was written by Dr ANM and all authors commented on previous versions of the manuscript. All authors read and approved the final manuscript.

Funding There is no funding to declare.

Data availability The data are not sensitive in nature.

\section{Declarations}

Conflict of interest The authors declared that they have no conflict of interest.

Ethical approval There is no ethical approval required.

\section{References}

Abeler J, Bäcker M, Buermeyer U, Zillessen H (2020) COVID-19 contact tracing and data protection can go together. JMIR mHealth uHealth 8(4):e19359

Ahmed W, Angel N, Edson J, Bibby K, Bivins A, O’Brien JW, Li J (2020) First confirmed detection of SARS-CoV-2 in untreated wastewater in Australia: A proof of concept for the wastewater surveillance of COVID-19 in the community. Sci Toalt Environ 728:138764

Alabool H, Alarabiat D, Abualigah L, Habib M, Khasawneh AM, Alshinwan M, Shehab M (2020) Artificial intelligence techniques for containment COVID-19 Pandemic: a systematic review

Bhalla N, Pan Y, Yang Z, Payam AF (2020) Opportunities and challenges for biosensors and nanoscale analytical tools for pandemics: COVID-19. ACS Nano 14(7):7783-7807

Bhowmick GD, Dhar D, Nath D, Ghangrekar MM, Banerjee R, Das S, Chatterjee J (2020) Coronavirus disease 2019 (COVID-19) outbreak: some serious consequences with urban and rural water cycle. Npj Clean Water 3(1):1-8

Bilal M, Nazir MS, Rasheed T, Parra-Saldivar R, Iqbal HMN (2020) Water matrices as potential source of SARS-CoV-2 transmission-an overview from environmental perspective. Case Stud Chem Environ Eng 2:100023

Ceylan Z (2020) Estimation of COVID-19 prevalence in Italy, Spain, and France. Sci Total Environ 729:138817

Chellaswamy C, Anumolu J, Kaliraja T, Polisetty NP (2019) cloud assisted waste water management system for smart cities. Paper presented at the 2019 Innovations in power and advanced computing technologies (i-PACT)

Cho H, Ippolito D, Yu YW (2020) Contact tracing mobile apps for COVID-19: privacy considerations and related trade-offs. arXiv preprintarXiv:2003.11511

Curl JM, Nading T, Hegger K, Barhoumi A, Smoczynski M (2019) Digital twins: the next generation of water treatment technology. J Am Water Works Ass 111(12):44-50

DHI (2020) The one tip for an efficient wastewater treatment plant: go digital. DHI Reservoir.

Farkas K, Hillary LS, Malham SK, McDonald JE, Jones DL (2020) Wastewater and public health: the potential of wastewater surveillance for monitoring COVID-19. Curr Opin Environ Sci \& Health. 17(14):20 
Ferretti L, Wymant C, Kendall M, Zhao L, Nurtay A, Abeler-Dörner L, Fraser C (2020) Quantifying SARS-CoV-2 transmission suggests epidemic control with digital contact tracing. Science. https://doi. org/10.1126/science.abb6936

Franch-Pardo I, Napoletano BM, Rosete-Verges F, Billa L (2020) Spatial analysis and GIS in the study of COVID-19 . A review. Sci Total Environ 739:140033

Garrido-Baserba M, Corominas L, Cortés U, Rosso D, Poch M (2020) The fourth-revolution in the water sector encounters the digital revolution. Environ Sci Technol 54(8):4698-4705

Han H, Zhu S, Qiao J, Guo M (2018) Data-driven intelligent monitoring system for key variables in wastewater treatment process. Chin J Chem Eng 26(10):2093-2101

Hart OE, Halden RU (2020) Computational analysis of SARS-CoV-2/ COVID-19 surveillance by wastewater-based epidemiology locally and globally: feasibility, economy, opportunities and challenges. Sci Total Environ 730:138875

Hart OE, Halden RU (2020) Simulated 2017 nationwide sampling at 13,940 major US sewage treatment plants to assess seasonal population bias in wastewater-based epidemiology. Sci Total Environ 727:138406

Hashem IAT, Ezugwu AE, Al-Garadi MA, Abdullahi IN, Otegbeye O, Ahman QO, Mbah GC, Shukla AK, Chiroma H (2020) A machine learning solution framework for combatting COVID-19 in smart cities from multiple dimensions. medRxiv

Kitajima M, Ahmed W, Bibby K, Carducci A, Gerba CP, Hamilton KA, Haramoto E, Rose JB (2020) SARS-CoV-2 in wastewater: state of the knowledge and research needs. Sci Total Environ 739:139076

Kolditz O, Rink K, Nixdorf E, Fischer T, Bilke L, Naumov D, Liao Z, Yue T (2019) Environmental information systems: paving the path for digitally facilitated water management (Water 4.0). Engineering 5(5):828-832

La Rosa G, Bonadonna L, Lucentini L, Kenmoe S, Suffredini E (2020) Coronavirus in water environments: occurrence, persistence and concentration methods-a scoping review. Water Res 179:115899

Medema G, Been F, Ruijgers H (2020) The SARS-Cov-2 sewage water samples of 29 July. KWR Water Research Institute

Morley J, Cowls J, Taddeo M, Floridi L (2020) Ethical guidelines for COVID-19 tracing apps. Nature Publishing Group, Berlin

Mounce SR (2020) Data science trends and opportunities for smart water utilities. In: ICT for smart water systems: measurements and data science. Springer, Cham, pp 1-26

Paleologos EK, O’ Kelly BC, Tang C, Cornell K, Rodríguez-Chueca J, Abuel-Naga H, Koda E, Farid A, Vaverková MD, Kostarelos K (2020) Post COVID-19 water and wastewater management to protect public health and geoenvironment. Environ Geotech 8(3): 193-207

Pan L, Mu M, Yang P, Sun Y, Wang R, Yan J, Li P, Hu C (2020) Clinical characteristics of COVID-19 patients with digestive symptoms in Hubei, China: a descriptive, cross-sectional, multicenter study. Am J Gastroenterol 115:766-773

Parker MJ, Fraser C, Abeler-Dörner L, Bonsall D (2020) Ethics of instantaneous contact tracing using mobile phone apps in the control of the COVID-19 pandemic. J Med Ethics 46(7):427-431
Poch M, Garrido-Baserba M, Corominas L, Perelló-Moragues A, Monclús H, Cermerón-Romero M, Melitas N, Jiang SC, Rosso D (2020) When the fourth water and digital revolution encountered COVID-19. Sci Total Environ 744:140980

Qureshi D, Salim M, Singh P, Swarnkar P, Goud H (2020) Robotics solutions to combat novel corona virus disease-2019 (COVID-19). In: Pushpendra and Swarnkar, Pankaj and Goud, Harsh, Robotics Solutions to Combat Novel Corona Virus Disease-2019 (COVID19)(June 2, 2020)

Rary E, Anderson SM, Philbrick BD, Suresh T, Burton J (2020) Smart sanitation-biosensors as a public health tool in sanitation infrastructure. Int J Environ Res Public Health 17(14):5146

Salathé M, Althaus CL, Neher R, Stringhini S, Hodcroft E, Fellay J, Zwahlen M, Senti G, Battegay M, Wilder-Smith A (2020) COVID19 epidemic in Switzerland: on the importance of testing, contact tracing and isolation. Swiss Med Weekly 150(11-12):20225

Sims N, Kasprzyk-Hordern B (2020) Future perspectives of wastewater-based epidemiology: monitoring infectious disease spread and resistance to the community level. Environ Int 139:105689

Street R, Malema S, Mahlangeni N, Mathee A (2020) COVID-19 wastewater surveillance: an African perspective. Sci Total Environ 743:140719

Suresh S, Renukappa S, Kamunda A (2019) Building information modelling in the framework of knowledge management: a water industry case study. In: Paper presented at the proceedings of the 2019 3rd international conference on information system and data mining

U.N.E.P (2020) COVID-19, wastewater, and sanitation. United Nation Environment Programme

W.H.O (2019) Water, sanitation, hygiene, and waste management for the COVID-19 virus. https://apps.who.int/iris/bitstream/handle/ 10665/331499/. World Health Organization, [Accessed July 2020]

W.H.O (2020) Water, sanitation, hygiene, and waste management for the COVID-19 virus: interim guidance, 23 April 2020: World Health Organization

W.R.C (2020) SARS-CoV-2 and wastewater. Water Research Commmission

Wang J, Shen J, Ye D, Yan X, Zhang Y, Yang W, Li X, Wang J, Zhang L, Pan L (2020) Disinfection technology of hospital wastes and wastewater: suggestions for disinfection strategy during coronavirus disease 2019 (COVID-19) pandemic in China. Environ Pollut 262:114665

Wilkinson A (2020) Local response in health emergencies: key considerations for addressing the COVID-19 pandemic in informal urban settlements. Environ Urban. https://doi.org/10.1177/09562 47820922843

Zhang N, Gong Y, Meng F, Bi Y, Yang P, Wang F (2020) Virus shedding patterns in nasopharyngeal and fecal specimens of COVID19 patients. medRxiv. 